$\Rightarrow$ VALVULAR DISEASE

Role of rivaroxaban after TAVI

\section{Current guidelines recommend dual} antiplatelet therapy early after transcatheter aortic valve implantation (TAVI) to prevent thromboembolic events. Given that this recommendation is based mainly on expert consensus, investigators in the GALILEO trial assessed an antithrombotic strategy with a low dose (10 mg daily) of the direct factor $\mathrm{Xa}$ inhibitor rivaroxaban compared with an antiplatelet-based strategy in patients without an established indication for oral anticoagulation after successful TAVI. However, the trial was terminated early owing to safety concerns. Results presented at the AHA Scientific Sessions 2019 indicate that a strategy with low-dose rivaroxaban is associated with a higher risk of death or thromboembolic events and a higher risk of bleeding than an antiplatelet-based strategy.

A total of 1,644 patients were randomly assigned to receive rivaroxaban $10 \mathrm{mg}$ daily (with aspirin 75-100 mg daily for the first 3 months) or aspirin 75-100 mg daily (with clopidogrel $75 \mathrm{mg}$ daily for the first 3 months). After a median of 17 months, the intention-totreat analysis showed that the rivaroxaban group had a higher incidence of death or a first thromboembolic event (105 versus 78 patients; HR 1.35, 95\% Cl 1.01-1.81, $P=0.04$ ) and of major, disabling or life-threatening bleeding events (46 versus 31 patients; HR 1.50, 95\% Cl 0.95-2.37, $P=0.08$ ) than the antiplatelet group.

By contrast, an imaging substudy of the trial indicates that the rivaroxaban-based strategy is more effective than antiplatelet therapy in preventing subclinical leafletmotion abnormalities. Among 231 patients who underwent $4 \mathrm{D} \mathrm{CT}$ evaluation at 90 days after randomization, reduced leaflet motion (grade $\geq 3$ ) in one or more leaflets was less frequent in the rivaroxaban group than in the antiplatelet group ( $2.1 \%$ versus $10.9 \%$ ), as was subclinical thickening of one or more leaflets (12.4\% versus $32.4 \%$ ). Nevertheless, the investigators caution that given the findings of the main trial they cannot recommend routine imaging for leaflet-motion evaluation or routine use of anticoagulation after TAVI for preventing leaflet-motion abnormalities. Irene Fernández-Ruiz

ORIGINAL ARTICLES Dangas, G. D. et al. A controlled trial of rivaroxaban after transcatheter aortic-valve replacement. N. Engl.J. Med. https://doi.org/10.1056/NEJMoa1911425 (2019)| De Backer, O. et al. Reduced leaflet motion after transcatheter aortic-valve replacement. N. Engl.J.Med. https://doi.org/10.1056/ NEJMoa1911426 (2019)

\title{
Early aortic valve surgery reduces mortality
}

Asymptomatic patients with very severe aortic stenosis (AS) who undergo early surgical aortic valve replacement are less likely to die from the procedure or from cardiovascular causes than patients receiving conservative care. This finding from the RECOVERY trial, presented at the AHA Scientific Sessions 2019, supports the early intervention with aortic valve replacement surgery in asymptomatic patients with very severe AS.

"Although one-third to one-half of patients with severe [AS] are asymptomatic at the time of diagnosis, appropriate timing of intervention for these patients remains controversial," explain the study investigators. The RECOVERY trial was a randomized, open-label study designed to compare the long-term outcomes of early aortic-valve replacement surgery with a conservative-care approach in asymptomatic patients with very severe AS. In total, 145 patients were enrolled and randomly assigned to early surgery $(n=73)$ or conservative care $(n=72)$. Aortic valve replacement surgery was successfully performed in all patients assigned to the early-surgery group, and no operative mortality was observed. Among the patients receiving conservative care, $74 \%$ underwent surgical or transcatheter aortic valve replacement during the follow-up period (a median of $\sim 6$ years for both groups).

In the intention-to-treat analysis, the primary end point (a composite of operative mortality and cardiovascular mortality) occurred in $1 \%$ of the early-surgery group and in $15 \%$ of the conservative-care group (HR 0.09, 95\% CI 0.01-0.67, $P=0.003$ ). Furthermore, the early-surgery group had a lower incidence of death from any cause than the conservative-care group ( $7 \%$ versus $21 \%$ ).

Together, these findings indicate that earlier intervention is associated with better outcomes for asymptomatic patients with very severe AS. Whether early surgery can also benefit those with less severe AS remains to be determined.

Karina Huynh

ORIGINAL ARTICLE Kang, D.-H. et al. Early surgery or conservative care for asymptomatic aortic stenosis. N. Engl. J.Med. https://doi.org/10.1056/NEJMoa1912846 (2019)

\section{Udenafil to FUEL exercise performance in individuals with Fontan physiology}

Udenafil improves measures of exercise performance at the ventilatory anaerobic threshold in individuals with Fontan physiology. This finding comes from the FUEL trial, which was presented at the AHA Scientific Sessions 2019.

In children born with single-ventricle congenital heart disease, the Fontan operation connects the inferior vena cava to the pulmonary artery. Individuals with this physiology usually experience a decline in cardiovascular efficiency and exercise performance during adolescence and into adulthood. Investigators in the phase III FUEL trial tested the use of udenafil, a phosphodiesterase type 5 inhibitor. A total of 400 participants with Fontan physiology (mean age 15.5 years) were recruited from 30 centres in North America and South Korea and were randomly assigned to receive udenafil (87.5 mg twice daily) or placebo.

The primary end point (between-group difference in change in $\mathrm{O}_{2}$ consumption at peak exercise) was not significantly different between the udenafil and placebo groups (+ $2.8 \%$ versus $-0.2 \% ; P=0.071$ ). However, analyses performed during submaximal exercise at the ventilatory anaerobic threshold indicated improvements in the udenafil group compared with the placebo group in $\mathrm{O}_{2}$ consumption, ventilatory equivalents of $\mathrm{CO}_{2}$ and work rate. No significant changes were observed in the myocardial performance index or in the serum level of B-type natriuretic peptide.

"These findings indicate that therapy with udenafil improves cardiovascular physiology at moderate levels of exercise in the cohort of patients who have undergone total cavopulmonary connection," conclude the investigators. "Ongoing surveillance is needed to determine the effect of chronic treatment with udenafil on the long-term clinical course of those living with single-ventricle congenital heart disease."

Gregory B. Lim

ORIGINAL ARTICLE Goldberg, D. J. et al. Results of the Fontan Udenafil Exercise Longitudinal (FUEL) trial. Circulation https://doi.org/10.1161/CIRCULATIONAHA.119.044352 (2019) 